\title{
An Introduction to a Library material management and security control system - Radio Frequency Identification (RFID) technology
}

\section{Thanuja C. Ranawella \\ B.Sc. Hons. (Kelaniya), MLS (Colombo), ASLLA Acting Librarian Kotalawala Defence Academy,}

\section{Abstract}

Radio Frequency Identification (RFID) allows an item, (i.e. library book) to be tracked and communicated with by using radio waves. There are several methods of identification, but the most common is to store a serial number that identifies a person or object, and perhaps other information, on a microchip that is attached to an antenna. In a library environment, RFID technology resembles a traditional barcode system in that it provides a means of assigning an ID to an item and reading that ID to perform circulation transactions or to take inventory. Introducing RFID, a library is able to identify and locate even the most remotely out of place item and helps to speed inventory process, and enhances the customer experience by increasing product availability and delivering faster checkouts. This allows the library to improve its circulation and inventory control, which helps to optimize the allocation of labour and financial resources. Selfservice checkout and check-in is enjoying greater and greater acceptance at libraries around the world. With no special item orientations required or scan line to find customers enjoy an extremely high self- service success rate. So our libraries can further increase the number of customers who use this time- and labour-saving technology. The driver for today's libraries to adopt RFID is the need to increase efficiency and reduce cost. Automation and self-service can help libraries of all sizes toward achieving these aims, and RFID has the added advantage that it can also provide security for the range of different media on offer in libraries.

\section{Introduction}

During the 1950s libraries moved from a card based circulation system to a computer based system (Cadoo, 2005). This improved privacy with borrowing details now being moved from the public arena (in circulation cards stored in pocket within specific items) to the safety of the library's databases (Schuyler, 2004, p. 22). With the adoption of barcode technology in the 1970's - 1980's many libraries were able to streamline workflow within circulation servers. The 
barcode allowed a quicker and efficient way to retrieve unique identifier, which assisted libraries to track items. However, barcodes need to be scanned within a handheld scanner (Lichtenberg, 2003, p.14) or within 'line of sight'.

In recent-years, the technology has received increased attention due to a confluence of events including technology advancement, heightened security concerns, supply chain automation, and a continuing emphasis on cost control within industries. Therefore RFID has found its way or is being tested in a number of different industries. These include tracking retail stocks, controlling individuals' access to building and even preventing counterfeiting of trademarked clothing. This technology is an automatic identification method, relying on storing and remotely retrieving data using devices called RFID tags or transponders. An RFID tag is a small object that can be attached to or incorporated into a product, animal, or person.. Passive tags require no internal power source, whereas active tags require a power source. These wireless automatic identification data capture systems allow for non-connected reading or writing of data and are highly effective in environments where barcode labels can not survive.

The use of RFID has now been extended to libraries so as to keep them efficient and competitive to face the new challenges in the always-changing workspace. It has been used in library circulation, to track books, videotapes, and other library collection components as well as item security very successfully.

The reliability of the system, its ease of operation, and the flexibility of tagging all kinds of media easily are important criteria in choosing an RFID system. There should be no need for an additional server, which means that products can be connected easily to a library's network. Interfaces to the most popular management systems worldwide also already exist.

Libraries are finding that RFID technology holds many advantages over traditional barcodes and antitheft tags, and contributes to realize considerable labour and cost savings.

\section{RFID Technology}

The concept of RFID technology is similar to that of a cellular phone. It is a combination of radio -frequency-based technology and microchip technology and uses radio waves to automatically identify people or objects. The information contained on microchips in the tags affixed to library materials is read using radio frequency technology regardless of item orientation or alignment (i.e., the technology does not require line-of-sight or a fixed plane to read tags as do traditional theft detection systems) and distance from the item is not a critical factor except in the case of extra-wide exit gates. The corridors at the building exit(s) can be as wide as four feet because the tags can be read at a distance of up to two feet by each of two parallel exit sensors. (Boss, 2004) 


\section{Components of an RFID System}

RFID system has four main components: (1) RFID tags that are electronically programmed with unique information; (2) readers or sensors to interrogate the tags (3) antenna; and (4) server.

\section{1) RFID Tags}

The heart of the system is the RFID tag. It is a paper thin electronic device that holds data, which is flexible and allows it to be placed inconspicuously on the inside, cover of each book or directly onto CDs and videos and covered with a property sticker in a library's collection. It consists of an etched antenna and a tiny silicon chip which stores vital bibliographic data including a unique ID number to identify each item and also store a security bit and if needed, information to support sorting system. These tags can store far more information about an item than a barcode, which has been the traditional approach to library automation. In addition, RFID chips are read/write, so the data in them can be updated whenever necessary. Having all this information readily available on the media chip means that, time-consuming data retrieval from databases can be omitted.

Another difference between RFID and barcodes is that barcodes require line-ofsight. A person must orient the barcode towards a scanner for it to be read. RFID chips, by contrast, do not require line-of-sight and can be read as long as they are within the range of a reader - a reading distance of approximately one foot is sufficient for a desktop reader. The benefit for librarians is less stress and less repetitive work.

RFID tags also allows the use of a single label for both circulation and security. When newly acquired media are entered into the collection, only one intelligent tag needs to be attached. This cuts manual effort per operation in half. RFID tags can be used for status control as well as for security checks. There is no need for an additional security stripe.

Finally, unlike in the case of barcodes pasted on the outer cover of a book an RFID tag can last longer as it is pasted inside. Most RFID vendors claim a minimum of 100,000 transactions before a tag may need to be replaced.

\section{2) Readers or sensors:}

This devices used at building exits are usually called "sensors." while the ones used for circulation and inventorying are usually called "readers" The reader powers an antenna to generate an RF field. When a tag passes through the field, the information stored on the chip in the tag is interpreted by the reader and sent to the server, which, in turn, communicates with the integrated library system when the RFID system is interfaced with it. 
Exit sensors verify that all material leaving the library has been checked out. Sensors at exits are basically two types. Some RFID systems have an interface between the exit sensors and the circulation software to identify the items moving out of the library. Were a library user to leave the library with a stolen book and not be caught, the library would at least know what had been stolen. This is done by designating a bit as the "theft" bit and turning it off at time of charge and on at time of discharge. If the material that has not been properly charged is taken past the exit gate sensors, an immediate alarm is triggered.

Another option is to use both the "theft" bit and the online interface to an integrated library system, the first to signal an immediate alarm and the second to identify what has been taken out. If the user card/ patron identification cards, also has an RFID tag, the library will also be able to determine, who removed the items without properly charging the materials and the persons who access restricted areas and services. This could be a better solution for the biggest problem of library material thefts.

RFID readers are used in the staff workstation of a library in the following manner.

\section{a)Conversion Station}

This is where library data are written to the tags. Using reader and portable cart you can quickly and easily convert library materials from traditional optical barcode technology to RFID technology. Therefore there is no need to connect it to your automated circulation system, a touch-sensitive screen, or an optical barcode scanner.

\section{b) Staff Workstation at Circulation:}

This workstation is designed especially for library staff to facilitate the smooth handling of books and other materials having RFID tags. Checking out, checking in, renewal, monitoring, sorting and admin functions can be done at this station.

After establishing the validity of a member card the books can be checked out of the library. Checkout station is used to process several items at the same time. A stack of books can be placed on the deck of this station, which will instantly read the RFID tag attached to the books while activating or de-activating their antitheft function.

The books returned by the borrowers are put on the deck of check in station by the library staff. Late fine if any can be automatically calculated and updated on the database. Simultaneously, the theft detection system automatically can be activated. Finally, a receipt is also printed out to confirm returning of borrowed material. The validity of the renewals is also checked and accordingly database is updated.

The use of RFID reduces the amount of time required to perform circulation operations. The most significant time savings are attributable to the facts that information can be read from RFID tags much faster than from barcodes and that several items in a stack can be read at the same time. Earlier, we had to scan barcode and charge/discharge anti theft detector separately but the RFID 
system combines barcode scanning and security into one operation, improving workplace efficiency and ergonomics. It processes both barcodes and RFID tags, and can perform smaller scale barcode-to-RFID conversions.

This technology helps librarians to reduce valuable staff time spent scanning barcodes while charging and discharging items and can also improve circulation, which helps allocate human and financial resources. This means that libraries can reduce their professional employees of routine work and operational tasks and they can be released for more productive and interesting duties. For the users, RFID speeds up the borrowing and return procedures.

\section{b) Self Service Units}

Using this technology books can be checked out independently by the borrower without any intervention of library staff. It makes circulation faster and easier. A borrower using self-charging finds it very convenient because he/she does not have to carefully place materials within a designated template and he/she can charge several items at the same time. This is a particular advantage to scientific libraries, where recently published books often experience a high demand.

The media are put on a desktop device that scans the information and updates the library's database with the checked-out status within milliseconds. The user receives a receipt with titles and due dates. This enables users to be totally independent of any staff. Self-discharging shifts the job of the staff to the patrons. For scientific libraries, this frees up more time for research and other tasks.

\section{d) Sorter and Conveyor:}

This is an automated system for returning material to proper area of library. The book is identified by a RFID reader unit as it is inserted into the return slot, and then placed into a bin. If this is connected to a multiple sorter in the background, then the books can be directly integrated into their systematic group or separated out if they are already marked as being on hold for another user. This significantly reduces the amount of staff time required to ready materials for reshelving and gives an opportunity for users to take a book immediately after being returned by another one.

3) Antenna: The antenna produces radio signals to activate the tag and read and write data to it. Antennas are the channels between the tag and the reader, which controls the system's data acquisitions and communication. The electromagnetic field produced by an antenna can be constantly present when multiple tags are expected continually. Antennas can be built into a doorframe to receive tag data from person's things passing through the door. 
4) Server: It is the communications gateway among the various components it receives the information from one or more of the readers and exchanges information with the circulation database. Its software includes the SIP/SIP2 (Session Initiation Protocol), APIs (Applications Programming Interface) NCIP (National Circulation Interchange Protocol) or SLNP necessary to interface it with the integrated library software but no library vendor has yet fully implemented NCIP approved by NISO (Koppel, 2004). The server typically includes a transaction database so that reports can be produced.

Optional RFID system includes the following three components. (Shahid, 2005)

\section{1) RFID Label Printer}

This is used to print the labels with an individual barcode, library logo, etc. When the print is applied, it simultaneously programs the data in to the chip. After this process, the RFID label is taken from the printer and applied to the book

\section{2) Handheld Reader/Inventory Wand}

Inventorying of the collection, which is a time consuming process, is made easier, quicker and accurate by using a portable handheld reader. This portable handheld reader can be moved rapidly across a shelf of books to read all of the unique identification information without somebody touching or removing them out to read the identification information for inventory control, search for books which are miss-shelved and search for an individual book requested. Previous systems relied on lists being corrected manually and then transferred to the database by hand. This means that a missing book or a misplaced item can be detected quickly automatically rather than handling each item individually. Collecting statistics is also simplified. For example, a librarian could determine how many times a serial collection is used.

\section{3) External Book Return}

An external book return is a machine with a slot with a chip RFID reader integrated into the wall which can offer a distinct service that is very useful for users, such as the ability to return books when the library is closed. It works the same way as the self-check in station. The user introduces himself/herself (if required by the library), and then puts the book(s) in to the slot. Upon completing the return, the user will receive a receipt showing how many and which books were returned. If this is connected to a multiple sorter in the background, then the books can be directly integrated into their systematic group or separated out if they are already marked as being on hold for another user. This return station also removes the book or other medium from the user's library account and reactivates the security function in the chip. 


\section{4) Book Drops}

Staff is relieved further when readers are installed in book drops. It could also be in the form of remote kiosks placed at various locations in the building/premises of the institute to enable patrons to drop the books for check- in to library of their own convenience without visiting library. External book return machine and book drops could be reduce the rate of overdue books and offers real-time check-in with round-the-clock flexibility for libraries with increasing circulation. Since they have already been checked-in, they can go directly back onto the shelves. These units can also be used with sorter and conveyor systems.

Implementing an RFID-system does not require existing user cards to be replaced. The parallel use of barcode cards or magnetic ID cards for identification is still possible. In such a situation, the RFID system supplier can provide an RFID-card with an interior design similar to the tag. Containing a programmable chip and an antenna, this ID card can carry much more information than the cards that were used previously. The chip card can be programmed with facilities such as access control to a lobby room or to Internet use. In addition, the card can include a payment function that enables use of, for example, a photocopier etc.

\section{Issues and questions with RFID Solutions}

According to the Boss (2004) and the software venders interviewed, following are the disadvantages.

- The major drawbacks of RFID technology is its cost. While the readers and gate sensors used to read the information typically cost around Rs. 1 million each, the tag costs around Rs. 50.00 each. These will discourage librarians from implementing a RFID system in their libraries. But librarians should be clever enough to find a funding agency to face the challenge. Otherwise they would not be able to be on par with new technologies.

- It is possible to compromise an RFID system by wrapping the protected material in two to three layers of ordinary household foil to block the radio signal. It is also possible to compromise an RFID system by placing two items against one another so that one tag overlays another. That may cancel out the signals. This requires knowledge of the technology and careful alignment.

- RFID tags are typically affixed to the inside back cover and are exposed for removal. This means that there would be problems when users become more familiar with the role of the tags. In Indian libraries, it is a major challenge to keep the tags intact.

- While the short-range readers used for circulation charge and discharge and inventorying appear to read the tags 100 percent of the time, the performance of the exit gate sensors is more problematic. They always don't 
read tags at up to twice the distance of the other readers. There is no library that has done a before and after inventory to determine the loss rate when RFID is used for security.

- Privacy concerns associated with item-level tagging is another significant barrier to library use of RFID tags. The problem with today's library RFID system is that the tags contain static information that can be relatively easily read by unauthorized tag readers. This allows for privacy issues described as "tracking" and "hotlisting". Tracking refers to the ability to track the movements of a book (or person carrying the book) by "correlating multiple observations of the book's bar code" or RFID tag. Hotlisting refers to the process of building a database of books and their associated tag numbers (the hotlist) and then using an unauthorized reader to determine who is checking out items in the hotlist.

- The signal from one reader can interfere with the signal from another in the same coverage.

\section{RFID in Libraries}

The most comprehensive application of RFID technology in libraries can be found in Singapore (Boss, 2003). Libraries in Singapore, under the leadership of the National Library Board, aggressively implemented RFID technology in their libraries. A very large percentage of the public libraries in Singapore are already using this technology with remarkable results. Libraries in United States, United Kingdom and India are also deploying with remarkable results (Boss 2004 and Klaus, 2003). Use of RFID technology in USA can be found in both public and academic libraries. New Hanover Counter Public Library in North Carolina and City Library at Santa Clara California were among the very early implementers of this technology.

Within the walis of modern Sri Lankan Libraries, thousands of books, periodicals, CD's and DVD's are contained within its inventory. Managing this inventory could be time consuming and very costly to the library administrators. Even though the use of the Dewey Decimal system and modern barcode technologies, one misplaced book could result in hours of wasted time and manpower to locate it due to the need for the book to actually be seen in order to identify it.

Burgeoning circulation and rising demand for new library services -without commensurate increases in budgets and staffing - are forcing many libraries to look to technology to help maintain personal service. Therefore the time has come to find alternatives for staff to fulfill the user requirement satisfactory and to implement better security and stock verification system for the libraries in Sri Lanka. But the knowledge about suitable new technologies, lack of experienced software developers locally and the budgetary constraints are main barriers for it. 
Most of the librarians interviewed have understood the importance of adopting new technologies in to their libraries because of high rate of misplacement and losses of library materials; increase in demand; and lack of staff. But some of them do not have sufficient knowledge about new technologies like RFID and others for example university librarians, though they have some knowledge do not have enough funds to implement such an expensive library management system.

However, the University of Moratuwa library was able to overcome the above barriers and was able to order the equipment to implement RFID system with the funds given by UGC and ADB and total cost of the project is around 5 million rupees for 50000 library materials. The Open University of Sri Lanka and Colombo Medical Faculty Libraries have decided to implement an RFID system for their libraries with the funds given by UGC, ADB and IRQUE (Improving Relevant Quality of Undergraduate Education Project) in the near future. They have already called quotations from software developers for the same.

The Library of Central Bank in Sri Lanka is also studying proposals to implement a security detection system, but they said they do not have enough allocation to implements a RFID library management system.

In the case of software developers of in Sri Lanka, none of them develops a library management system using RFID technology. Out of the 20 software developers 13 said that they were unable to do a RFID system for a library. Although the other 07 do not have experience of using RFID technology for libraries, they said they could give the above facilities for libraries with the use of RFID technology.

\section{Conclusion}

The main aim for modern libraries in adopting RFID is the need to increase efficiency and to have better service. Automation and self-service can help libraries of all sizes achieve these aims, and RFID has the added advantage that it can also provide security for the range of different media offered in libraries. The flexibility of tagging all kinds of media easily, multiple item check-in and checkout and easy convenience in operation are important criteria in choosing an RFID system.

The literature review was mainly used to identify the advantages as well as disadvantage of the RFID system in the context of library application. Informal conversations and face to face discussions were had with responsible officers of 20 software developers in Sri Lanka. Rapid charging and discharging, Simplified Self charging and discharging, High Reliability, High Speed Inventorying, 
Automated Material Handling, Tag Life were identified as key features of a RFID system according to the Software developers interviewed and the literature review.

In conclusion it is justifiable to state that Libraries in Sri Lanka do not implement the installment of RFID systems in their libraries mainly due to the lack of funds, and most of the librarians especially the public libraries do not have the knowledge of such kind of system. Therefore this paper suggests that libraries should be funded by the funding agencies such as Asia Development Bank, Improving Relevant Quality of Undergraduate Education Project etc. Responsible organizations should organize seminars with the help of software developers to educate the librarians on RFID system.

\section{Reference}

Boss R.W. (2003), RFID Technology for Libraries Library Technology Report November - December 2003

Boss R.W. (2004) RFID Technology for Libraries, ALA American Library Association

Cadoo Scott (2005), RFID use within libraries: an Australian perspective. Presented at the New Librarians' Symposium 2 Adelaide, Australia, 3-4 December 2004.

htt://conferences.alia.org.au/newlibrarian2004/zobjects/presymppapers/Cadoo websitepaperfinal.pdf

Klaus Finkenzeller. RFID Handbook John Wiley and Sons, 2003

Lichtenberg, James. (2003). Industry explores viability of RFID. Publishers Weekly, 205(46),2-3. Retrieved March 7, 2004, from EBSCO Academy search elite.

Mats G Lindquist,(2003) TFID in Libraries: introduction to issues, paper presented at the $69^{\text {th }}$ IFLA General Conference and Council, Berlin, 1-9 August 2003.http://www.ifla.org/IV/ifla69/papers/16le-Lindquist.pdf

Matthew Artz, (2003) City Library Adopts Controversial RFID Chips, http://www.berkeleydaily.org/article.cfm?issue $=10-10-03 \&$ storylD $=17547$

Raisisn an RFID Ruckus, Steve Ulfelder, October 7, 2003 http://www. ifla.org/IV/ifla69/papers/132e-Chachra.pdf 
Schuyler, Michael. (2004), RFID: Helpmate or conspiracy? Computers in libraries, 24 (1), 22-24, Retrieved March 7, 2004 from htt://gateway.library.qut.edu.au:2083/startftv.asp?source=ext\&EBSCOConte nt=ZWJjY8Lr7XePprZrvNfxa6Gmr3

Ron Harris, (2003) SF Library wants to Track Books with Computer Chips Associated Press, http://www.usatoday.com/tech/news/internetorivacy/200310-03-sf-library-rfid x.htm

Shahid Syed (2005). Use of Library Technology in Libraries: a New Approach to Circulation, Tracking, Inventorying, and Security of Library Materials Library Philosophy and Practice Vol. 8, No. 1 pp. 23-40 